\title{
Development of a novel necroptosis-associated miRNA risk signature to evaluate the prognosis of colon cancer patients
}

\author{
Yang Huang $^{1 \#}$, Yuanyuan Zou ${ }^{1 \#}$, Qiru Xiong ${ }^{1}$, Chao Zhang ${ }^{2}$, José María Sayagués ${ }^{3}$, Vishal G. Shelat ${ }^{4}$, \\ Xingyu Wang ${ }^{5}$ \\ ${ }^{1}$ General Surgery Department, The Second Affiliated Hospital of Anhui Medical University, Hefei, China; ${ }^{2}$ General Surgery Department, The First \\ Affiliated Hospital of Anhui Medical University, Hefei, China; ${ }^{3}$ Servicio de Anatomía Patológica, Hospital Clínico Universitario de Salamanca e \\ Instituto de Investigación Biomédica de Salamanca (IBSAL), Universidad de Salamanca, Salamanca, Spain; ${ }^{4}$ Department of General Surgery, Tan \\ Tock Seng Hospital, Singapore, Singapore; ${ }^{5}$ Emergency Surgery Department, The First Affiliated Hospital of Anhui Medical University, Hefei, \\ China \\ Contributions: (I) Conception and design: Y Huang, Y Zou; (II) Administrative support: X Wang; (III) Collection and assembly of data: Y Zou, Q \\ Xiong; (IV) Data analysis and interpretation: Y Huang, C Zhang; (V) Manuscript writing: All authors; (VI) Final approval of manuscript: All authors. \\ "These authors contributed equally to this work and should be considered as co-first authors. \\ Correspondence to: Yang Huang. General Surgery Department, The Second Affiliated Hospital of Anhui Medical University, 678 Feicui Road, Hefei \\ 230601, China. Email: huangyang@ahmu.edu.cn.
}

Background: Necroptosis is a recently discovered caspase-independent form of cell death which plays an important role in the occurrence and development of cancer. As an important regulatory factor in necroptosis, microRNAs (miRNAs) are important for the development of colon cancer. This study established a novel necroptosis-related miRNA risk signature to evaluate the prognosis of patients with colon adenocarcinoma (COAD).

Methods: The necroptosis-related miRNAs were selected by assessing the differential expression of miRNAs in 459 COAD patient samples and 8 control samples from The Cancer Genome Atlas (TCGA). Selection operator Cox analyses and survival analyses were used to establish the risk signature of 7 miRNAs related to necroptosis. Functional enrichment analysis and nomograms were used to explore the potential effects of necroptosis-related miRNAs on prognosis and metastasis. The target genes of the necroptosisrelated miRNAs were predicted using online databases and the genes related to overall survival (OS) were screened.

Results: The risk signature was based on 7 necroptosis-related miRNAs. Nomograms showed that the risk signature was effective at predicting the prognosis and TNM stage of COAD patients. Gene ontology (GO) and Kyoto Encyclopedia of Genes and Genomes (KEGG) analyses demonstrated that these miRNAs play an important role in cancer development, metastasis, and prognosis. A total of 38 target genes for these miRNAs were found to be associated with the OS in COAD patients.

Conclusions: This study provided novel evidence that necroptosis-related miRNAs are associated with the prognosis of COAD patients. A risk signature established based on these miRNAs could effectively predict the prognosis and metastasis of COAD in patients.

Keywords: Colon cancer; necroptosis-related miRNAs; prognosis; prognostic model

Submitted Oct 21, 2021. Accepted for publication Dec 17, 2021.

doi: 10.21037/atm-21-6576

View this article at: https://dx.doi.org/10.21037/atm-21-6576 


\section{Introduction}

Colon adenocarcinoma (COAD) is among the most common tumor of the digestive system. At present, COAD is the fourth most common cancer type in the United States and is the second leading cause of cancer-related mortality (1). In China, COAD is also one of the top five leading causes of cancer and cancer-associated deaths. Furthermore, the incidence of COAD has risen substantially over the past two decades, surpassing the rate of increase for rectal cancer (2). While the specific mechanisms of COAD remain to be fully elucidated, its incidence has been associated with dietary and genetic factors, inflammatory bowel disease, and diverticulosis $(3,4)$. Currently, the main forms of treatments include surgery, chemotherapy, and immunotherapy. However, within 5 years of treatment, an estimated 30 $50 \%$ of COAD patients ultimately develop recurrent or metastatic disease (5). Therefore, it is vital to develop novel approaches for diagnosing and evaluating the prognosis of COAD patients, thereby facilitating improved individualized treatment regimens.

Necroptosis is a recently identified form of caspaseindependent regulated cell death (6). Necroptosis has been linked to a range of inflammatory and malignant diseases including ischemic, cardiovascular, and cerebrovascular diseases, as well as cancers $(7,8)$. Studies have shown that necroptosis can inhibit tumor proliferation, metastasis, and progression (9), while also promoting reactive oxygen species (ROS) production and the cancer microenvironment (10-12). However, the mechanisms by which necroptosis promote tumorigenesis and progression in COAD remain to be fully elucidated.

MicroRNAs (miRNAs) are small transcripts of about 19-24 nucleotides long (13). Recent studies have found that although miRNAs lack coding ability, they can bind to tumor-related genomic regions or vulnerable sites in the genome, thereby regulating the expression of tumor suppressor genes and oncogenes (14). Moreover, particular miRNAs can influence oncogenesis by regulating necroptotic cell death. Visalli et al. found that miR-371-5p, miR-373, and miR-543 were upregulated in hepatocellular carcinoma (HCC) tissues compared to adjacent noncancerous tissues. These miRNAs bound to their target casp-8 mRNA transcripts, thereby promoting necroptosis of HCC cells (15). Another study suggested that miR-381$3 p$ may be a biomarker for predicting the sensitivity of cells to apoptosis and necroptosis and may be a potential therapeutic target for renal cell carcinoma (RCC) (16).
Cai et al. found that miR-148a-3p was up-regulated in acute pancreatitis and its deficiency inhibited necrosis, AP progression and inflammatory infiltration while inducing apoptosis in vivo (17).

Given the existing findings, we know that necroptosis plays an important role in the development of tumours and antitumour processes; however, its specific functions in COAD have been less studied. This present study utilized miRNA sequencing data from The Cancer Genome Atlas (TCGA) to define necroptosis-related miRNA expression profiles associated with COAD to define novel biomarkers with potential clinical and/or therapeutic relevance.

We present the following article in accordance with the REMARK reporting checklist (available at https://dx.doi. org/10.21037/atm-21-6576).

\section{Methods}

\section{Data sources}

The TCGA database was used to obtain the miRNA-seq data of patients with COAD. Mature miRNA sequences in the Fasta format were obtained from miRbase and utilized for miRNA annotations. The miRNA-seq data pertaining to 467 patient samples (459 tumors and 8 control samples) were analyzed. In addition, patient data including age, gender, stage, TNM classification, survival outcome, and survival duration were collated for analysis (Table 1). The study was conducted in accordance with the Declaration of Helsinki (as revised in 2013).

\section{Identification of differentially expressed necroptosis- associated miRNAs}

Based on prior review studies, 16 miRNAs known to be associated with necroptosis (18) were examined (Table S1). The expression of these miRNAs in COAD tumors and normal tissue samples was compared using the $\mathrm{R}$ 'reshape2' and 'limma' packages. Associations among necroptosisassociated miRNAs were assessed using Pearson correlation coefficients and the R 'ggplot2' package.

\section{Generation of the prognostic necroptosis-associated miRNA model}

The association between necroptosis-related miRNA expression and overall survival (OS) in patients was examined using the TCGA-COAD cohort. Univariate 
Table 1 Characteristics of colon adenocarcinoma patients in the The Cancer Genome Atlas (TCGA) database

\begin{tabular}{|c|c|}
\hline Variable & TCGA $(n=459)$ \\
\hline Male & 243 \\
\hline Female & 216 \\
\hline \multicolumn{2}{|c|}{ Age at diagnosis } \\
\hline$>65$ years & 269 \\
\hline \multicolumn{2}{|l|}{ Stage } \\
\hline I & 76 \\
\hline ॥ & 178 \\
\hline Unknown & 11 \\
\hline \multicolumn{2}{|l|}{ T stage } \\
\hline Tis & 1 \\
\hline $\mathrm{T} 1$ & 11 \\
\hline $\mathrm{T} 2$ & 78 \\
\hline T3 & 313 \\
\hline $\mathrm{T} 4$ & 56 \\
\hline \multicolumn{2}{|l|}{$\mathrm{N}$ stage } \\
\hline M1 & 65 \\
\hline Unknown & 57 \\
\hline
\end{tabular}

Stage: a combination of T, N and M stages is used to determine colon cancer Stage; I: the cancer cells grow into the second or third layer of the colon wall, and there are no cancer cells in the nearby lymph nodes or the extracolonic area; II: the cancer cells have grown to or beyond the fourth layer of the colon wall, and there are no cancer cells in the nearby lymph nodes or outside the colon; III: the cancer has spread from the colon to nearby lymph nodes or there is tumor deposition. Tumor deposits are small tumors in the fat around the colon. IV: the cancer has spread beyond the colon and to nearby lymph nodes; Tis: carcinoma in situ; T1: tumor invasion into the submucosa; T2: the tumor invaded the muscularis propria of the intestinal wall; T3: the tumor penetrated into the muscularis propria and invaded subserosal layer, or when the primary lesion was located in the colon and rectum without serosal layer, the tumor had invaded paracolonic or rectal tissues; T4: the tumor has penetrated the peritoneum or directly invaded other organs; N0: no metastasis to regional lymph nodes; N1: 1-3 regional lymph node metastases; N2: $\geq 4$ regional lymph node metastases; M0: no distant metastasis; M1: there are distant metastases. 
and multivariate Cox regression analyses were performed with a $\mathrm{P}$ value $<0.01$ as the cutoff criteria. Analyses were performed with the $\mathrm{R}$ 'survival' and 'forestplot' packages. Kaplan-Meier curves were utilized for survival analyses, with log-rank tests and univariate Cox proportional hazard regression models used to generate p-values as well as hazard ratios (HRs) with 95\% confidence intervals (CIs). Those necroptosis-associated miRNAs that exhibited prognostic relevance in the initial analyses were retained for further assessment. A risk score was defined based on these prognostic miRNAs using the TCGA dataset following centralization and standardization with the $\mathrm{R}$ 'scale' function. Risk scores were calculated using the following formula: risk score $=\sum 7 \mathrm{iXi} \times \mathrm{Yi}$, where $\mathrm{X}$ and $\mathrm{Y}$ correspond to coefficients and miRNA expression levels, respectively. The median risk scores were used to stratify patients from the TCGA-COAD cohort into low- and high-risk groups, after which OS was compared between these groups using the Kaplan-Meier approach. The 1and 5 -year OS of patients were analyzed using receiver operating characteristic (ROC) curves generated with the R 'survival, 'ROCR', and 'timeROC' packages. The prognostic relevance of necroptosis-associated miRNAs was further assessed through Cox regression analyses. KaplanMeier analyses were conducted as above and prognostic miRNAs were chosen for subsequent evaluation.

\section{Prognostic analyses}

Demographic and clinical data, including patient age, gender, and TNM staging, were extracted from the TCGA dataset. Univariate and multivariate Cox regression models were used to analyze the prognostic value of these variables and the risk scores generated above. Cox regression analysis was subsequently used to create prognostic and metastatic nomograms, which were validated using the consistency index (C-index) and calibration plots in the "Rms" $\mathrm{R}$ package v5.1.

\section{$R N A$ isolation and quantitative real-time polymerase chain reaction ( $q R T-P C R)$}

Total cellular RNA was isolated from colon cancer cell lines (SW480, SW620, HCT116, and LOVO) and a human colon tissue cell line (CCD18-Co) using Trizol reagent (Invitrogen, Carlsbad, CA, USA) and reverse transcribed into cDNA using a cDNA synthesis kit (Takara, Dalian, China) according to the manufacturers' instructions. qPCR amplification was performed using the SYBRGreen PCR Kit (Takara) in the ABI 7900 Sequence Detection System (Applied Biosystems, USA). The primers are shown in Table S2. U6 was used as the internal control. The PCR parameters were set for an initial cycle of $95{ }^{\circ} \mathrm{C}$ for 2 minutes, followed by 40 cycles at $95^{\circ} \mathrm{C}$ for 30 seconds, $57^{\circ} \mathrm{C}$ for 45 seconds, and $72{ }^{\circ} \mathrm{C}$ for 45 seconds. The relative expression of each gene was analyzed using the $2^{\Delta \Delta \mathrm{Ct}}$ method. Experiments were performed in duplicates and repeated 3 times.

\section{miRNA target gene enrichment analysis}

Putative target genes associated with prognostic miRNAs were identified using the TargetScan, miRDB, and miRTarBase databases. Genes that were predicted by at least two of these three databases were chosen as miRNA target genes. A miRNA and target gene interaction network were generated using Cytoscape (19), while gene ontology (GO) and Kyoto Encyclopedia of Genes and Genomes (KEGG) enrichment analyses were conducted using the $\mathrm{R}$ software.

\section{Statistical analysis}

Single-factor analysis of variance was applied to compare the gene expression levels between the normal ovarian and OC tissues. To assess the independent prognostic value of the risk model, we used univariate and multivariate Cox regression models. All statistical analyses were performed on SPSS v22.0. All of the experiments in our study were independently performed in triplicate, $\mathrm{P}<0.05$ was considered statistically significant.

\section{Results}

\section{Differentially expressed necroptosis-related miRNAs in COAD}

The expression of 16 necroptosis-associated miRNAs was compared in 459 tumor tissue samples and 8 normal healthy samples using the TCGA-COAD cohort. A total of 7 miRNAs were significantly differentially expressed (all $\mathrm{P}<0.001$; Table S3). The expressions of hsa-miR-141$3 \mathrm{p}$, hsa-miR-148a-3p, hsa-miR-16-5p, hsa-miR-200a5p, hsa-miR-223-3p, hsa-miR-425-5p, and hsa-miR-7$5 \mathrm{p}$ were increased in tumor tissues compared to normal healthy tissues (Figure 1A). Furthermore, there were significant positive correlations among these miRNAs (Figure 1B). Univariate regression analyses showed that four 
A

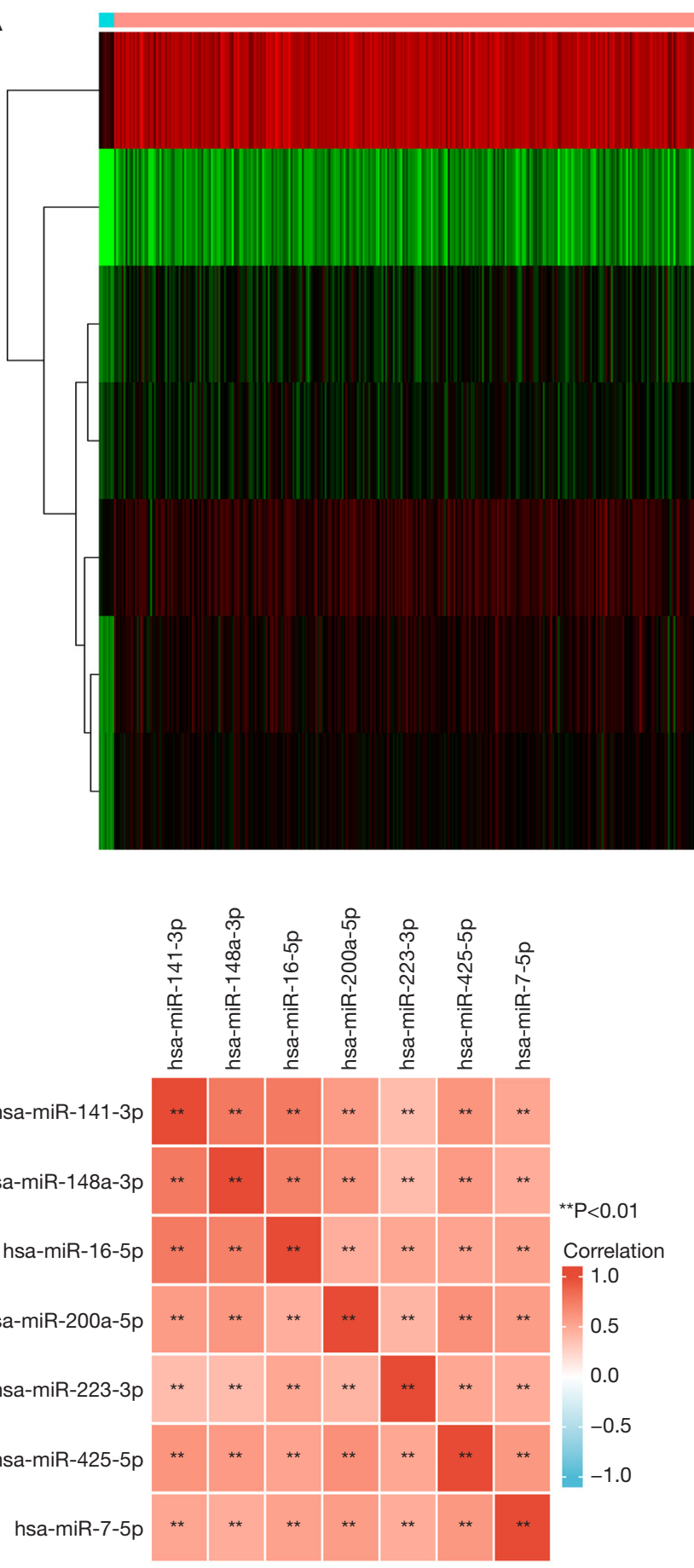

Type

hsa-miR-148a-3p

10

hsa-miR-7-5p

hsa-miR-223-3p

hsa-miR-425-5p

hsa-miR-200a-5p

hsa-miR-141-3p

hsa-miR-16-5p

C

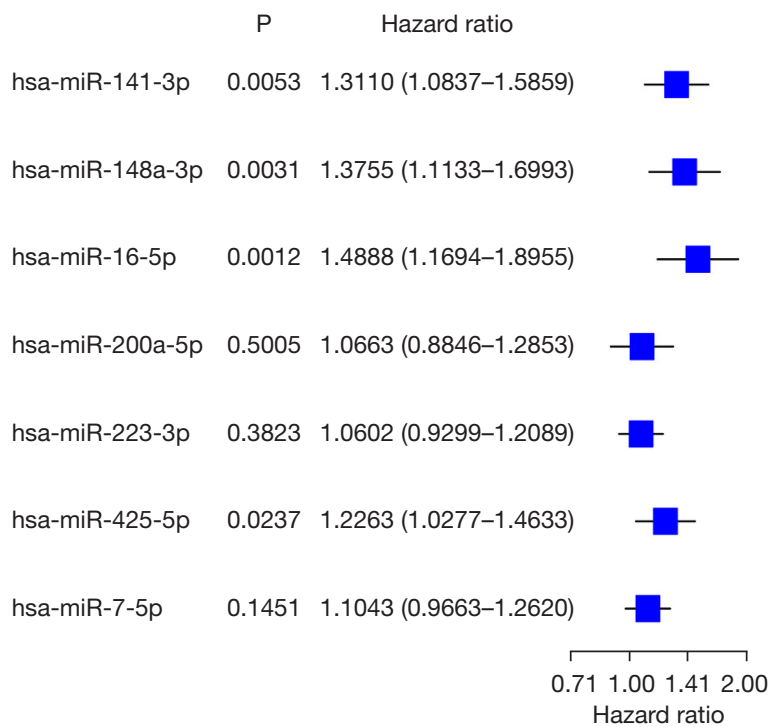

Figure 1 Analysis of the prognostic relevance of seven necroptosis-associated miRNAs. (A) Necroptosis-associated miRNA levels were compared between normal samples (N, blue) and tumor tissues ( $T$, pink). Green and red correspond to low and high levels of expression, respectively. (B) Heat maps were used to evaluate the correlation between different necroptosis-associated miRNAs in COAD patient samples. (C) Hazard ratios (95\% CI) and significance values for necroptosis-associated miRNAs that were related to patient OS. miRNA, microRNA; COAD, colon adenocarcinoma; CI, confidence interval; p, FDR-adjusted P value. 
Table 2 Univariate regression analyses of necroptosis-associated miRNAs

\begin{tabular}{lcc}
\hline Necroptosis-associated miRNA & Hazard ratio & P value \\
\hline hsa-miR-141-3p & 1.3110 & 0.0053 \\
hsa-miR-148a-3p & 1.3755 & 0.0031 \\
hsa-miR-16-5p & 1.4888 & 0.0012 \\
hsa-miR-200a-5p & 1.0663 & 0.5005 \\
hsa-miR-223-3p & 1.0602 & 0.3823 \\
hsa-miR-425-5p & 1.2263 & 0.0237 \\
hsa-miR-7-5p & 1.1043 & 0.1451 \\
\hline
\end{tabular}

Table 3 Multivariate Cox regression analyses of necroptosis-associated miRNAs

\begin{tabular}{lcc}
\hline Necroptosis-associated miRNA & Hazard ratio & P value \\
\hline hsa-miR-16-5p & 1.2810 & 0.1708 \\
hsa-miR-200a-5p & 0.7969 & 0.0717 \\
hsa-miR-223-3p & 0.5974 & 0.9555 \\
hsa-miR-425-5p & 1.0555 & 0.6984 \\
hsa-miR-141-3p & 1.0793 & 0.6244 \\
hsa-miR-7-5p & 1.0414 & 0.6494 \\
hsa-miR-148a-3p & 1.2917 & 0.1177 \\
\hline
\end{tabular}

of these necroptosis-associated miRNAs were significantly correlated with poor OS (HR >1; Figure 1C; Tables 2,3).

\section{Risk-related miRNA identification}

Considering the potential prognostic value of some necroptosis-associated miRNAs, experiments were conducted to identify the risk-related miRNAs that may be able to predict outcomes in COAD patients. Kaplan-Meier analyses revealed that elevated expression of hsa-mir-16-5p, hsa-mir141-3p, and hsa-mir-148a-3p were significantly correlated with decreased survival of patients (Figure $2 A-2 C$ ). This suggested that these miRNAs may play a deleterious role in the context of COAD-related necroptosis such that patients who express high levels of these miRNAs are likely to experience inferior oncologic outcomes.

\section{Development of the prognostic necroptosis-associated miRNA model}

Risk scores were calculated by using the following prognostic miRNA model: risk score $=(1.0793 \times$ hsa-miR$141-3 p$ exp $)+(1.2917 \times$ hsa-miR-148a-3p exp $)+(1.2810 \times$ hsa-miR-16-5p exp $)+(0.7969 \times$ hsa-miR-200a-5p exp $)+$ $(0.9555 \times$ hsa-miR-223-3p exp $)+(1.0555 \times$ hsa-miR-425$5 \mathrm{p} \exp )+(1.0414 \times$ hsa-miR-7-5p exp). The median risk scores were then used to stratify COAD patients into lowand high-risk cohorts. High-risk patients were found to have significantly poorer OS compared to low-risk patients $(\mathrm{P}=0.00004$; Figure $3 A)$. The ROC curve revealed that the $\mathrm{AUC}$ values for the 1 - and 5 -year survival rates were 0.659 and 0.656 , respectively (Figure $3 B$ ).

\section{Univariate and multivariate analyses of the risk model}

Univariate and multivariate Cox regression analyses were conducted to clarify whether the risk scores were independent predictors of patient prognosis. In univariate analyses, risk scores, $\mathrm{pT}$ stage, and $\mathrm{pN}$ stage were associated with COAD patient prognosis. Multivariate analysis showed that after adjusting for potential confounding variables, age, stage, and risk scores were independent predictors 

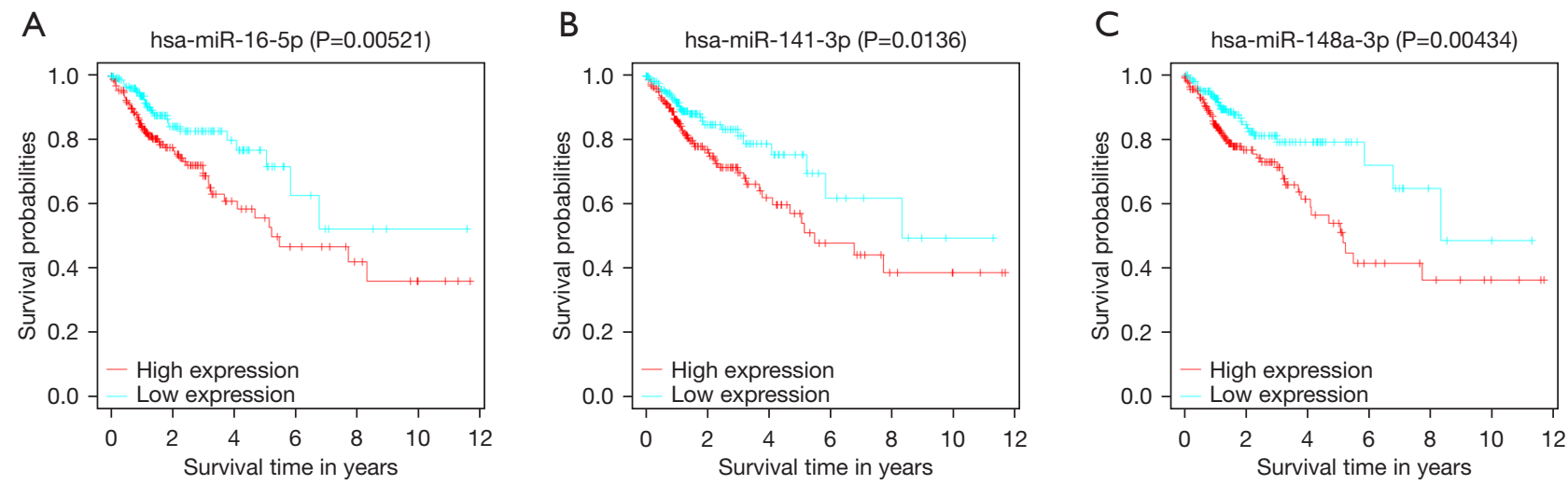

Figure 2 Assessment of COAD risk-related miRNAs. The overall survival of COAD patients expressing low and high levels of (A) hsa-miR16-5p, (B) hsa-miR-141-3p, and (C) hsa-miR-148a-3p. COAD, colon adenocarcinoma; miRNA, microRNA.

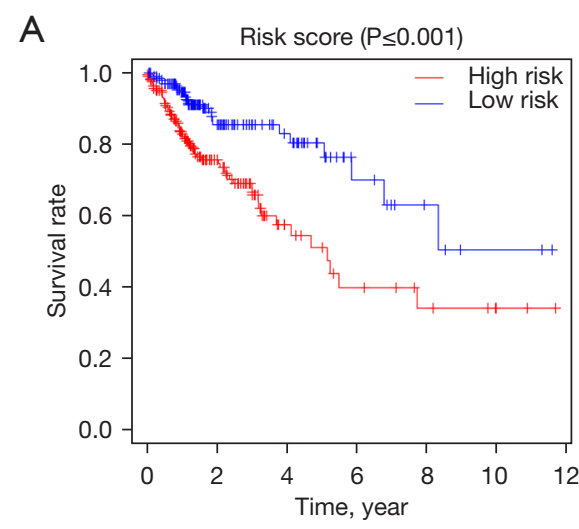

C

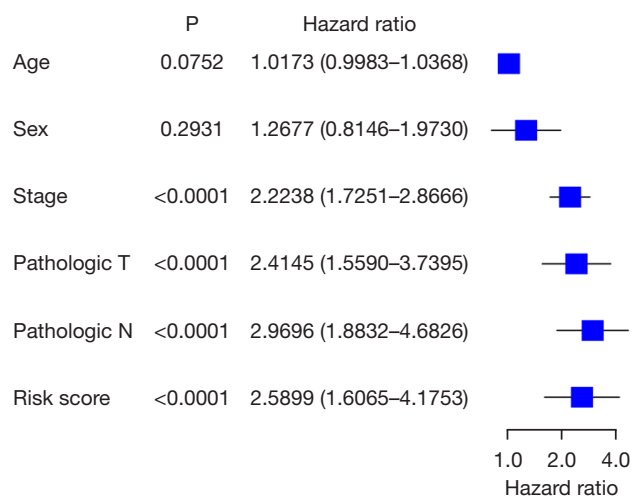

B

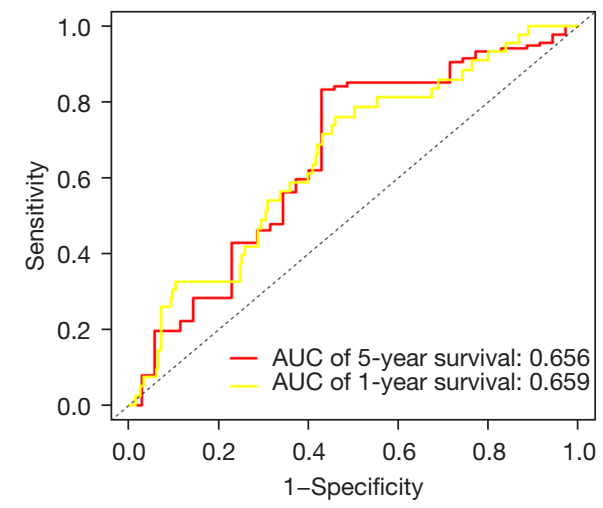

D

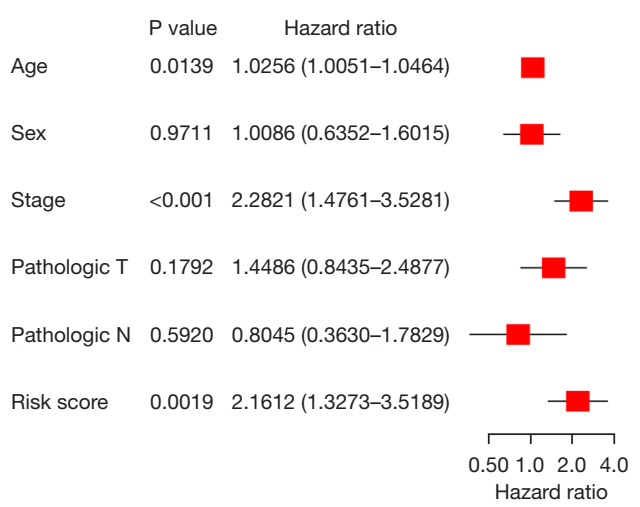

Figure 3 Development of the prognostic necroptosis-associated miRNA model. (A) The OS of high- and low-risk patients. (B) ROC curves demonstrating the prognostic value of the risk scores. (C) Univariate and (D) multivariate Cox analyses screening for variables with a significant correlation with OS. miRNA, microRNA; OS, overall survival; ROC, receiver operating characteristic; AUC, area under the curve. 
Table 4 Univariate regression analyses of risk factors in colon cancer

\begin{tabular}{lcc}
\hline Clinicopathological parameters & Hazard ratio & P value \\
\hline Age & 1.0173 & 0.0752 \\
Gender & 1.2677 & 0.2931 \\
Stage & 2.2238 & $<0.0001$ \\
Pathologic_T & 2.4145 & $<0.0001$ \\
Pathologic_N & 2.9696 & $<0.0001$ \\
Risk score & 2.5899 & $<0.0001$ \\
\hline
\end{tabular}

Table 5 Multivariate Cox regression analyses of risk factors in colon cancer

\begin{tabular}{lcc}
\hline Clinicopathological parameters & Hazard ratio & P value \\
\hline Age & 1.0256 & 0.0139 \\
Gender & 1.0086 & 0.9711 \\
Stage & 2.2821 & $<0.001$ \\
Pathologic_T & 1.4486 & 0.1792 \\
Pathologic_N & 0.8045 & 0.5920 \\
Risk score & 2.1612 & 0.0019 \\
\hline
\end{tabular}

of prognosis in COAD patients $(\mathrm{HR}=2.1612,95 \% \mathrm{CI}$ : 1.3273 to $3.5189 ; \mathrm{HR}=1.0256,95 \% \mathrm{CI}: 1.0051$ to 1.0464 ; and $\mathrm{HR}=2.2821,95 \% \mathrm{CI}: 1.4761$ to 3.5281 , respectively) (Figure 3C,3D; Tables 4,5). Moreover, the expression of these 7 miRNAs was significantly elevated in colon cancer cell lines compared to normal colon cells (Figure 4A-4G).

\section{Development of the necroptosis-associated miRNA nomogram models}

Nomograms for OS at 1-, 3-, and 5-year were created based on age, stage, pathologic $\mathrm{T}$ stage, and risk scores (Figure 5A). The variable score is based on the intersection of the vertical line and point axis associated with each variable, and the total risk score is the sum of the scores of each variable. These total scores identify predictors of OS for each patient. The C-index of the nomogram was 0.772 , indicating that the predicted results were generally consistent with the actual OS of COAD patients. Finally, the calibration plots visually demonstrated that the predicted outcomes of the nomograms were in strong agreement with the actual prognostic outcomes at 1-, 3-, and 5-year (Figures 5B-5D).

\section{Evaluation of miRNA target genes}

The TargetScan, miRDB, and miRTarBase databases were used to predict the putative target genes of the hsa-miR16-5p, hsa-miR-141-3p and hsa-miR-148a-3p These three miRNAs were statistically significant in the prognosis of COAD. Target genes that overlapped in different databases were further analyzed (Figure $6 A$ and available online: https://cdn.amegroups.cn/static/public/atm-216576-01.pdf). The association between these miRNAs and their target genes were assessed with Cytoscape (Figure 6B). GO enrichment analyses of these target genes revealed that they were enriched in biological processes (BPs) including mitotic cell cycle, dephosphorylation, deubiquitination, and epithelial cell proliferation. They were also enriched for molecular function terms (MFs) including protein serine/threonine kinase activity and transforming growth factor-beta receptor binding, as well as cellular component terms (CCs) including recycling endosome, protein kinase complex, transferase complex, transferring phosphorus-containing groups, focal adhesion, and cell-substrate junction (Figure $6 \mathrm{C}$ and available online: https://cdn.amegroups.cn/static/ 

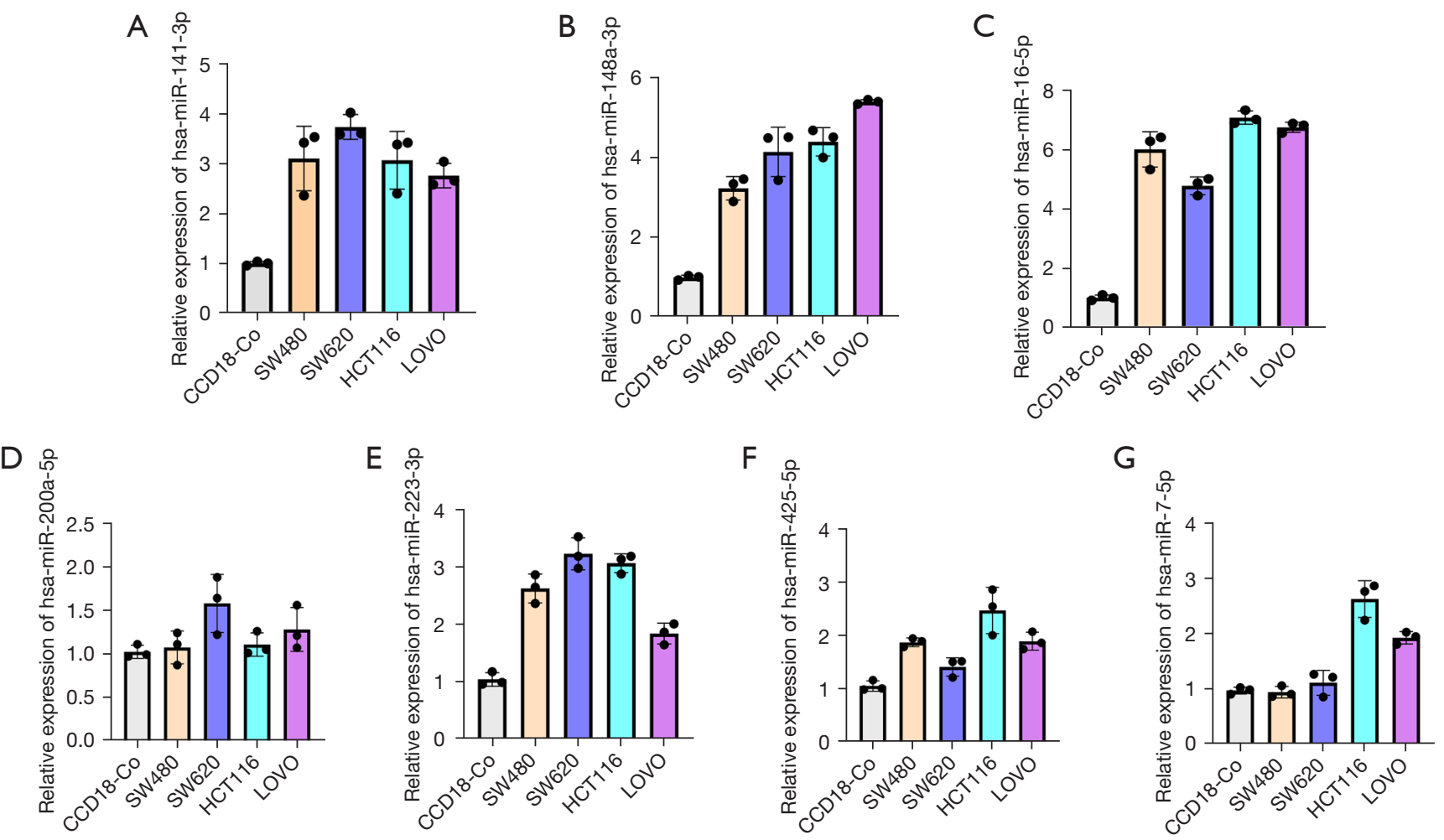

Figure 4 The expression levels of 7 miRNAs in the colon cancer cell lines SW480, SW620, HCT116, and LOVO, and the human colon tissue cell line CCD18-Co.

public/atm-21-6576-02.pdf). KEGG pathway enrichment analyses indicated that these target genes were enriched in the MAPK, mTOR, FOXO, p53, and PI3K-Akt signaling pathways (Figure $6 D$ and available online: https://cdn. amegroups.cn/static/public/atm-21-6576-03.pdf).

\section{Analysis of the prognostic relevance of miRNA target genes}

Analysis of the prognostic relevance of the miRNA target genes revealed significant associations between the expression of 38 genes and the OS of COAD patients (Figure 7 and Figure S1).

\section{Discussion}

Necroptosis is a form of regulated cell death that is often considered to be secondary to apoptosis in a range of pathological conditions (20). During cell necroptosis, the volume of the cell increases, the organelles expand, ultimately leading to the rupture of the plasma membrane and cell death $(21,22)$. Interestingly, necroptosis can promote the occurrence and progression of tumors by triggering a pro-oncogenic tumor immune microenvironment $(12,23,24)$ and modulating stromal cell responses $(25,26)$. However, in certain cases, necroptosis can inhibit tumor progression. Indeed, experimental studies on tumor immunology have found that necroptosis may play an important role in triggering immunogenicity and promoting natural or treatment driven anti-cancer immune monitoring $(27,28)$.

Several necroptosis-associated miRNAs have been suggested to play important roles in various cancers (29). In glioblastoma, mesenchymal glioblastoma subtypes exhibit extensive necrosis, and low miR-218 expression is a characteristic feature of the highly necrotic and hypoxic mesenchymal glioblastoma subset (30). Furthermore, the expression of miR-193a-3p is significantly reduced in malignant pleural mesothelioma, and it has been shown to regulate necrotic cell death by targeting the apoptosisrelated gene MCL-1 (31).

This current study evaluated the expression of 16 miRNAs associated with necroptosis and demonstrated that 7 miRNAs were upregulated in COAD tumor tissues compared with normal colon tissues. In addition, there was 
A

Points

Age

Stage

Pathologic T

Risk score

Total points

1 -year OS

3-year OS

5 -year OS
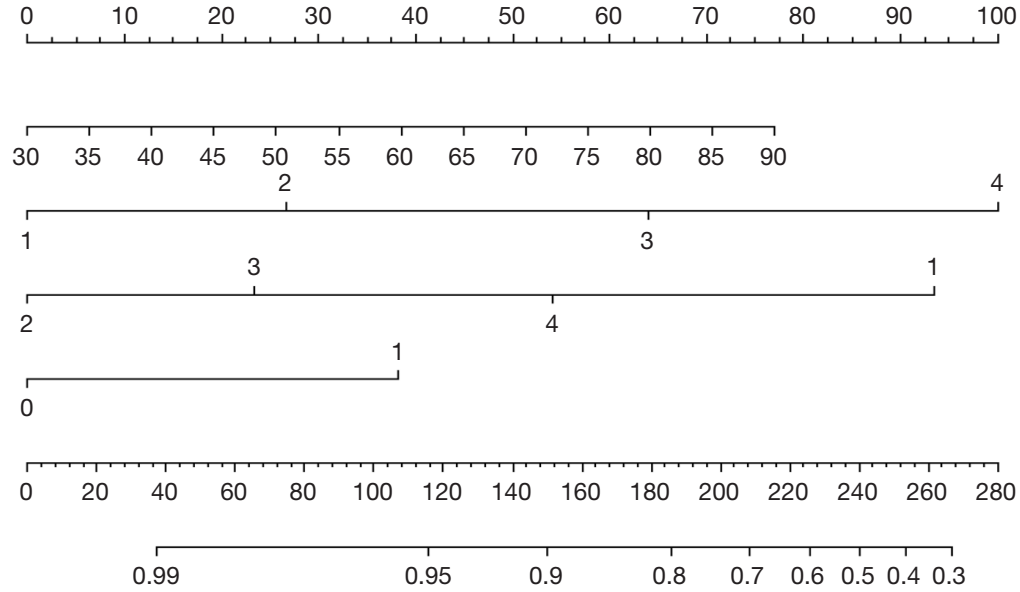

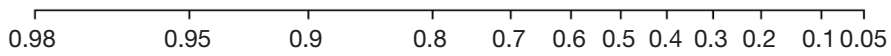

\begin{tabular}{llllllllllllll}
\hline 0.98 & 0.95 & 0.9 & & 0.8 & 0.7 & 0.6 & 0.5 & 0.4 & 0.3 & 0.2 & 0.10 .05 & 0.01
\end{tabular}
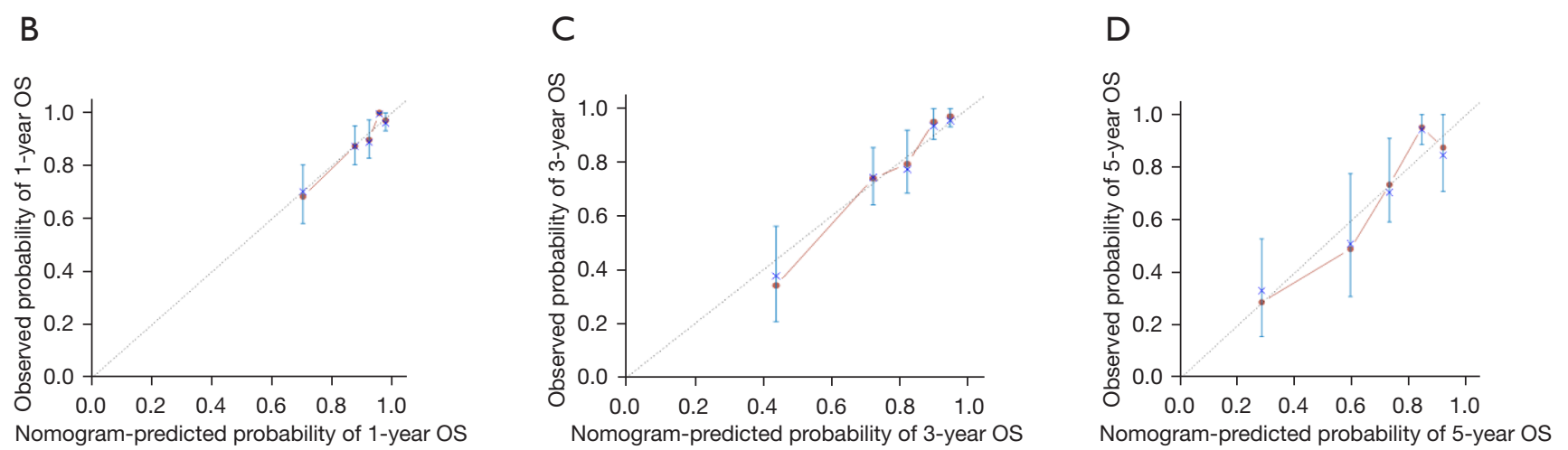

Figure 5 Construction of prognostic nomograms based on necroptosis-related miRNAs. (A) Nomograms predicting of the 1-, 3-, and 5-year OS. Calibration plots of the nomogram to predict the probability of the (B) 1-year, (C) 3-year, and (D) 5-year OS. miRNA, microRNA; OS, overall survival.

a significant positive correlation among the 7 miRNAs, suggesting that these may work together to regulate necroptosis in COAD. A risk score and prognostic model based on the 7 miRNAs was constructed and shown to accurately predict the 1 - and 5-year OS in COAD patient. These risk scores, together with patient age and tumor stage, were found to be independently associated with COAD patient prognosis. Other studies have also explored factors that are predictive of COAD prognosis. Zhou et al. confirmed the role of immune-related prognostic models in COAD (32). Autophagy-associated prognostic features have also been identified as reliable predictors of COAD patient prognosis (33). Our novel necroptosis-associated miRNA- based model may represent a valuable tool for predicting prognosis and survival in COAD patients.

To further evaluate the prognostic value of these necrosis-related miRNAs, univariate regression analyses were performed. The miRNAs hsa-miR-141-3p, hsa-miR$148 \mathrm{a}-3 \mathrm{p}$, and hsa-miR-425-5p were correlated with the prognosis of COAD patients. However, other researchers have reported that upregulation of hsa-miR-141-3p inhibited the growth of colon tumors (34). Li et al. showed that in necrotizing enterocolitis, the expression of hasmiR-141-3p can inhibit ripk1-mediated necrosis, thereby protecting small intestinal epithelial cells from injury (35). Upregulation of miR-148a-3p in gliomas has been 
A

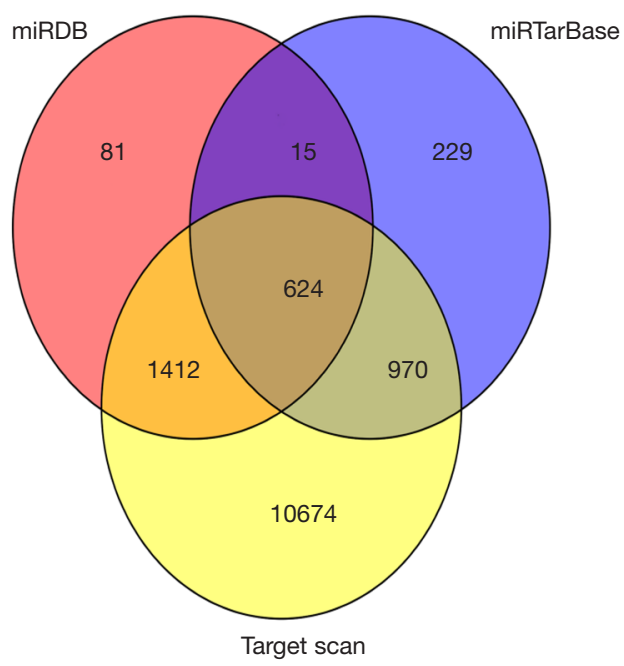

C

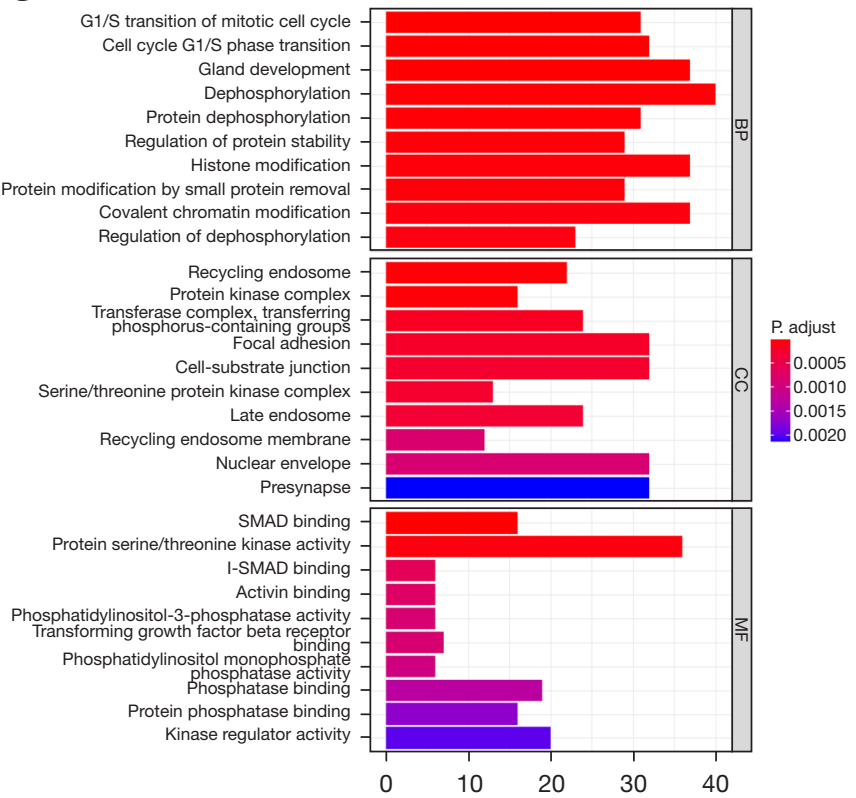

B
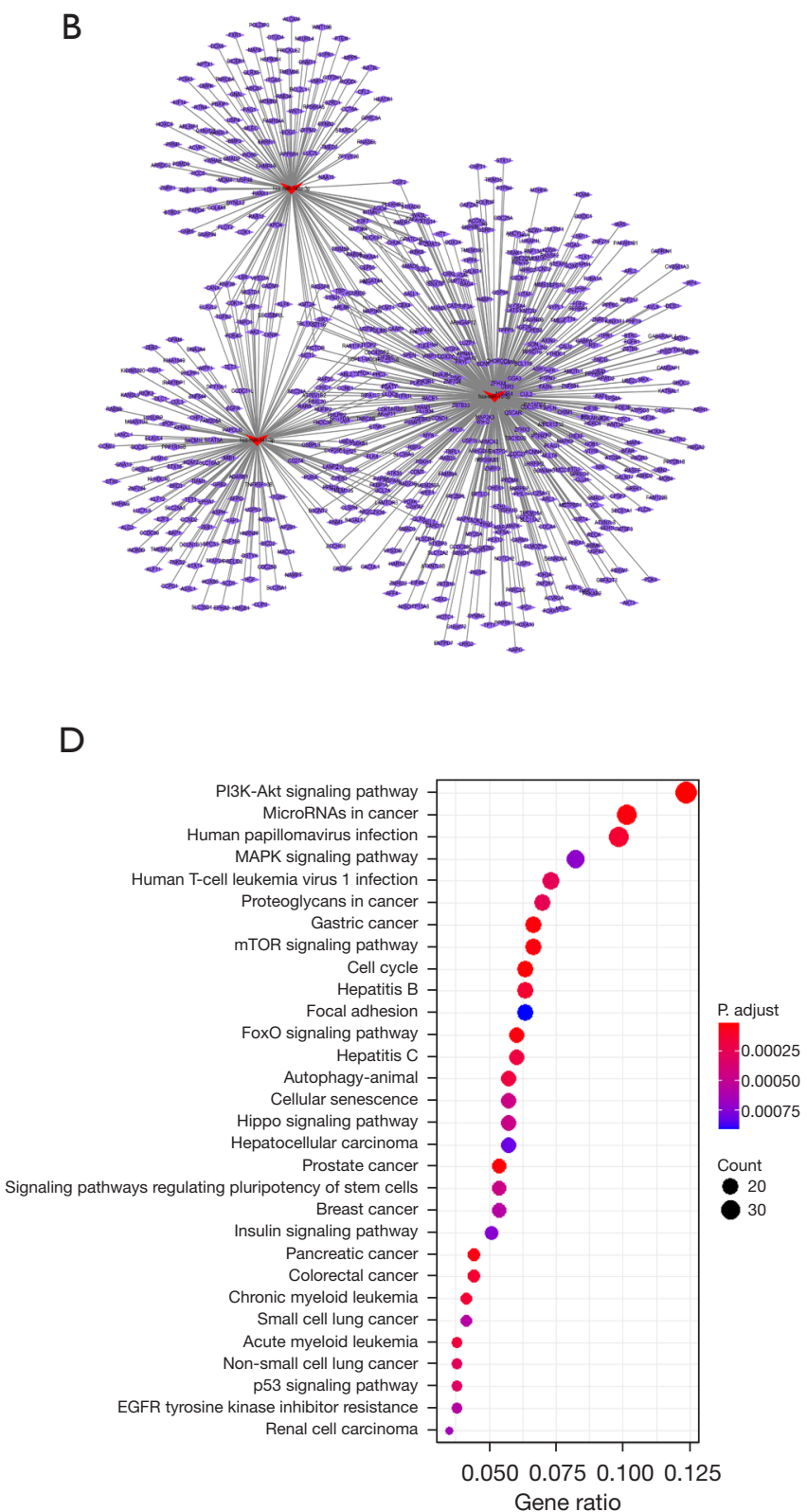

Figure 6 Analysis of miRNA target genes. (A) Intersecting miRNA target genes were identified through analyses of three databases. (B) Potential associations among the miRNAs and the target genes were assessed with Cytoscape. (C) The GO term enrichment analysis assessing BP, MF, and CC. (D) The KEGG enrichment analysis. miRNA, microRNA; GO, gene ontology; KEGG, Kyoto Encyclopedia of Genes and Genomes; BP, biological processes; MF, molecular functions; CC, cellular components.

associated with accelerated progression and poor survival outcomes (36). miR-148a-3p has also been found to be upregulated in distant metastatic tissue from osteosarcoma patients, and its upregulation is inversely correlated with distant metastasis, tumor size, and OS (37). In line with our findings, Kjersem et al. reported that plasma miR-148a expression was associated with decreased progression free survival (38). Previous studies have suggested that miR425-5p may promote the development and metastasis of colorectal cancer by activating the CTNND1-mediated $\beta$-catenin pathway (39). In sepsis, the miR-425-5p/RIP1 axis can repair related liver injury through necroptosis 
A

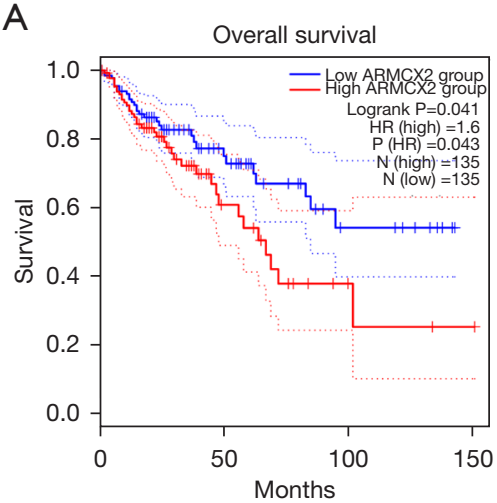

D

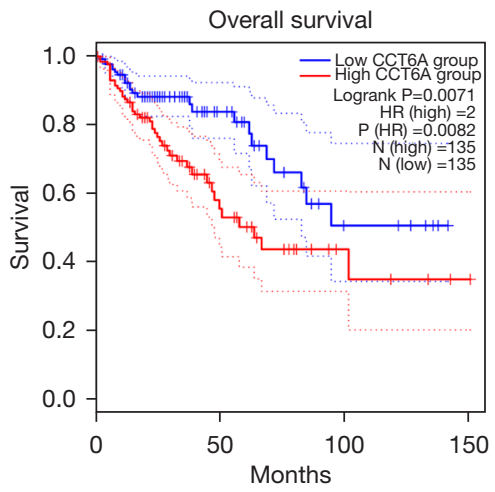

G

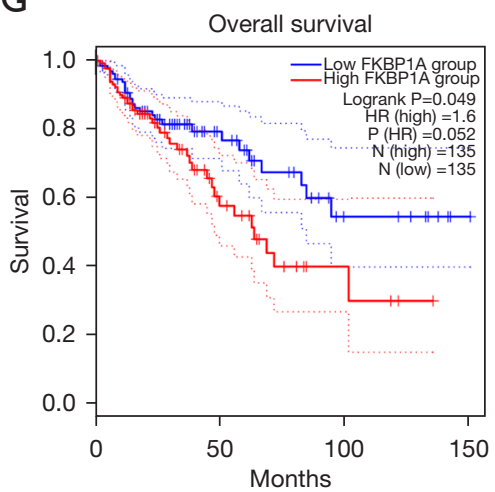

B

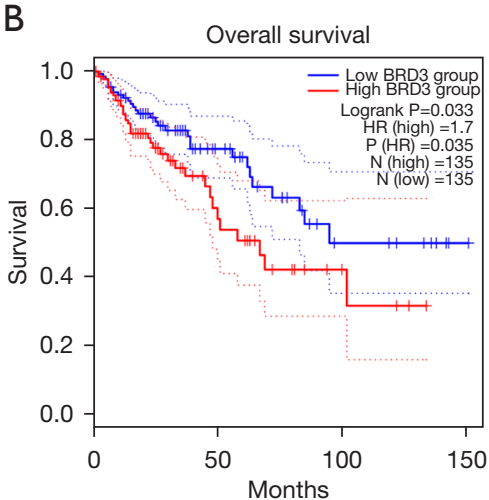

E

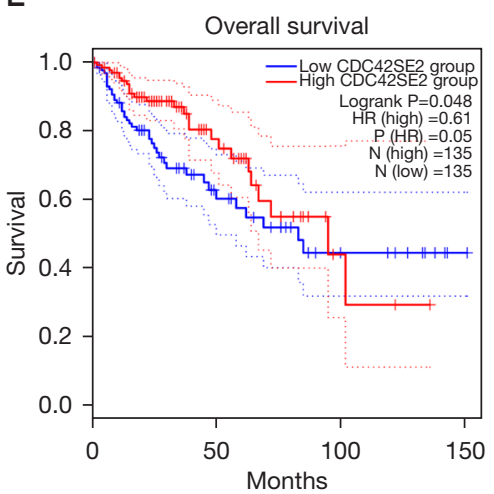

$\mathrm{H}$

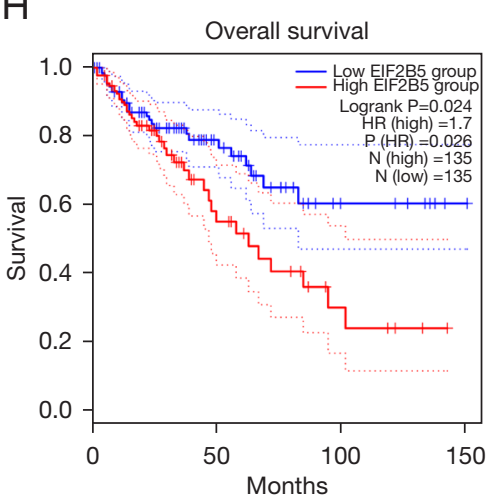

C

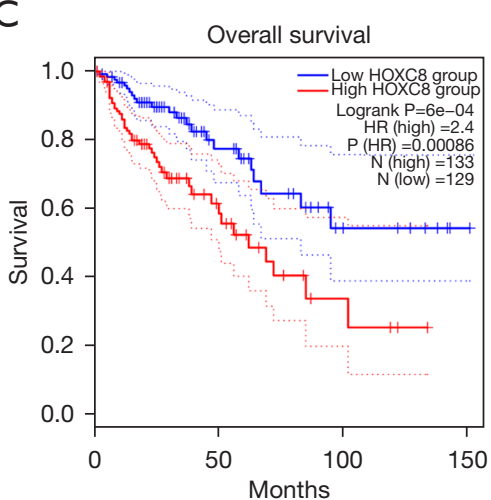

F

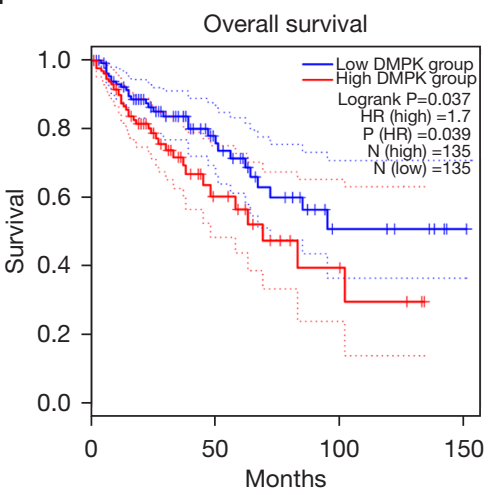

I

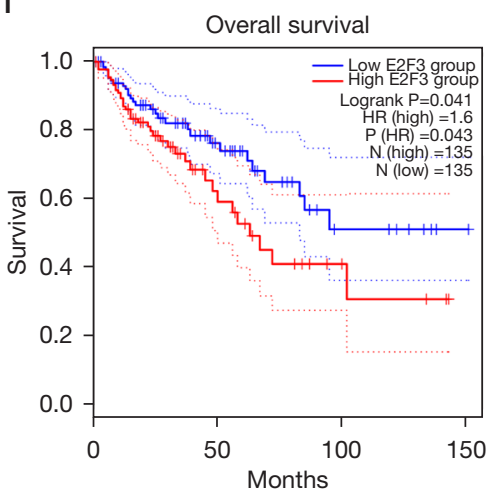

Figure 7 Analysis of the relationship between COAD patient OS and expression of the differentially expressed miRNA target genes. COAD, colon adenocarcinoma; OS, overall survival; miRNA, microRNA.

and inflammation (40). We found that ha-miR-16-5p was highly expressed in colon cancer and was associated with poor prognosis of colon cancer. Interestingly, previous studies have found that the expression and role of mir16 in cancer are uncertain. Hasáková et al. found that the expression level of mir-16 was up-regulated in colon cancer tissues compared with normal tissues (41). It has also been found that miR-16 was down regulated in colon cancer and was associated with late TNM stage, poor histological grade, and better OS $(42,43)$.

Our study confirmed that risk signatures can effectively classify COAD patients into subgroups with different risk levels. The risk signatures were combined with clinical factors to construct a new nomogram to predict 
the prognosis and TNM stage of COAD patients. The nomograms and c-index values were effective at predicting clinical outcomes. The validity of nomogram model was also confirmed by calibration curve analysis. Thus, this current study established models that can effectively predict the clinical outcomes of OS and TNM stage in COAD patients. Compared to previous prognostic models for colon cancer, this prediction model used a novel approach to assess the OS of COAD patients.

The potential target genes of the differentially expressed necroptosis-related miRNAs were identified and functional analyses were conducted. In KEGG pathway analyses, these target genes were found to be enriched in the FOXO, p53, Hippo, PI3K/Akt/mTOR, MAPK, ErbB, AMPK, apelin, RAS, PD-L1, TGF- $\beta$, focal adhesion, and stem cell pluripotency regulatory signaling pathways. The PI3K/Akt/ mTOR pathway is a key regulator of metastatic progression and chemoresistance in colorectal cancer (44). Hu and colleagues reported that $\mathrm{PI} 3 \mathrm{~K}$ can promote necroptotic cell death by interacting with RIP3 (45). Similarly, p53 can promote necroptotic cell death by upregulation of apoptosisinducing factors, interacting with Bak, and directly binding to Bcl-XL and Bcl-2 (46). The Hippo signaling pathway is an important regulator of colon cancer but its role in the context of necrotic cell death remains unclear (47). Kim et al. reported the ability of bee venom to disrupt focal adhesion and the integrity of the cell membrane, inducing necroptotic pluripotent stem cells death (48). The apelin signaling pathway is related to the invasion and migration of colon cancer cells. While its association with necroptosis in COAD has not been fully eludicated, it may be a promising anticancer therapeutic target (49). Furthermore, a link between necroptotic cell death and immune checkpoint activity has been identified. Lomphithak et al. reported a positive correlation between necroptosis and the expression of PD-L1. The researchers found that high expression of PD-L1 was correlated with longer patient survival (50). Additional investigations are needed to verify this in COAD. Target gene-based survival analyses revealed that 38 of these genes were associated with COAD patient OS. High expression of 20 of these genes was associated with poor survival outcomes, while elevated expression of 16 of these genes was associated with improved patient prognosis. Therefore, these target genes may be involved in cancer development and may be potential biomarkers for the prognosis and treatment of patients with COAD.

In conclusion, the results of this analysis suggested that the occurrence and progression of COAD are closely related to necroptosis. There are some limitations to this research. This study was conducted using the TCGACOAD data set, and other data sets should be similarly analyzed to validate our findings. Moreover, future in vitro and in vivo experiments are warranted to support the clinical findings.

\section{Conclusions}

This investigation identified new necroptosis-related miRNA markers capable of predicting the prognosis of patients with colon cancer. The knowledge obtained from this study could aid precision medicine in routine clinical practice.

\section{Acknowledgments}

The authors appreciate the academic support from the AME Colorectal Cancer Collaborative Group. Funding: None.

\section{Footnote}

Reporting Checklist: The authors have completed the REMARK reporting checklist. Available at https://dx.doi. org/10.21037/atm-21-6576

Conflicts of Interest: All authors have completed the ICMJE uniform disclosure form (available at https://dx.doi. org/10.21037/atm-21-6576). The authors have no conflicts of interest to declare.

Ethical Statement: The authors are accountable for all aspects of the work in ensuring that questions related to the accuracy or integrity of any part of the work are appropriately investigated and resolved. The study was conducted in accordance with the Declaration of Helsinki (as revised in 2013).

Open Access Statement: This is an Open Access article distributed in accordance with the Creative Commons Attribution-NonCommercial-NoDerivs 4.0 International License (CC BY-NC-ND 4.0), which permits the noncommercial replication and distribution of the article with the strict proviso that no changes or edits are made and the original work is properly cited (including links to both the formal publication through the relevant DOI and the license). See: https://creativecommons.org/licenses/by-nc-nd/4.0/. 


\section{References}

1. Siegel RL, Miller KD, Jemal A. Cancer statistics, 2020. CA Cancer J Clin 2020;70:7-30.

2. Wu C, Li M, Meng H, et al. Analysis of status and countermeasures of cancer incidence and mortality in China. Sci China Life Sci 2019;62:640-7.

3. O'Keefe SJ. Diet, microorganisms and their metabolites, and colon cancer. Nat Rev Gastroenterol Hepatol 2016;13:691-706.

4. Nakahara R, Amano Y, Murakami D, et al. Relationship between colonic diverticulosis and colon neoplasms in Japanese patients. Dig Endosc 2021;33:418-24.

5. Schmoll HJ, Van Cutsem E, Stein A, et al. ESMO Consensus Guidelines for management of patients with colon and rectal cancer. a personalized approach to clinical decision making. Ann Oncol 2012;23:2479-516.

6. Galluzzi L, Kroemer G. Necroptosis: a specialized pathway of programmed necrosis. Cell 2008;135:1161-3.

7. Xie Y, Zhao Y, Shi L, et al. Gut epithelial TSC1/mTOR controls RIPK3-dependent necroptosis in intestinal inflammation and cancer. J Clin Invest 2020;130:2111-28.

8. Ji X, Wang R, Tang H, et al. Necroptosis of osteoblasts was induced by breast cancer cells in vitro. Transl Cancer Res 2020;9:500-7.

9. Wang T, Jin Y, Yang W, et al. Necroptosis in cancer: An angel or a demon? Tumour Biol 2017;39:1010428317711539.

10. Tafani M, Sansone L, Limana F, et al. The Interplay of Reactive Oxygen Species, Hypoxia, Inflammation, and Sirtuins in Cancer Initiation and Progression. Oxid Med Cell Longev 2016;2016:3907147.

11. Weinlich R, Oberst A, Beere HM, et al. Necroptosis in development, inflammation and disease. Nat Rev Mol Cell Biol 2017;18:127-36.

12. Seifert L, Werba G, Tiwari S, et al. The necrosome promotes pancreatic oncogenesis via CXCL1 and Mincleinduced immune suppression. Nature 2016;532:245-9.

13. Calin GA, Sevignani C, Dumitru CD, et al. Human microRNA genes are frequently located at fragile sites and genomic regions involved in cancers. Proc Natl Acad Sci U S A 2004;101:2999-3004.

14. Wu ZH, Zhong Y, Zhou T, et al. miRNA biomarkers for predicting overall survival outcomes for head and neck squamous cell carcinoma. Genomics 2021;113:135-41.

15. Visalli M, Bartolotta M, Polito F, et al. miRNA expression profiling regulates necroptotic cell death in hepatocellular carcinoma. Int J Oncol 2018;53:771-80.
16. Zhao C, Zhou Y, Ran Q, et al. MicroRNA-381$3 p$ Functions as a Dual Suppressor of Apoptosis and Necroptosis and Promotes Proliferation of Renal Cancer Cells. Front Cell Dev Biol 2020;8:290.

17. Cai SW, Han Y, Wang GP. miR-148a-3p exhaustion inhibits necrosis by regulating PTEN in acute pancreatitis. Int J Clin Exp Pathol 2018;11:5647-57.

18. Liu Y, Chen Q, Zhu Y, et al. Non-coding RNAs in necroptosis, pyroptosis and ferroptosis in cancer metastasis. Cell Death Discov 2021;7:210.

19. Shannon P, Markiel A, Ozier O, et al. Cytoscape: a software environment for integrated models of biomolecular interaction networks. Genome Res 2003;13:2498-504.

20. Molnár T, Mázló A, Tslaf V, et al. Current translational potential and underlying molecular mechanisms of necroptosis. Cell Death Dis 2019;10:860.

21. Zong WX, Thompson CB. Necrotic death as a cell fate. Genes Dev 2006;20:1-15.

22. Vandenabeele P, Galluzzi L, Vanden Berghe T, et al. Molecular mechanisms of necroptosis: an ordered cellular explosion. Nat Rev Mol Cell Biol 2010;11:700-14.

23. Wegner KW, Saleh D, Degterev A. Complex Pathologic Roles of RIPK1 and RIPK3: Moving Beyond Necroptosis. Trends Pharmacol Sci 2017;38:202-25.

24. Jiao D, Cai Z, Choksi S, et al. Necroptosis of tumor cells leads to tumor necrosis and promotes tumor metastasis. Cell Res 2018;28:868-70.

25. Tortola L, Nitsch R, Bertrand MJM, et al. The Tumor Suppressor Hace1 Is a Critical Regulator of TNFR1Mediated Cell Fate. Cell Rep 2016;15:1481-92.

26. Strilic B, Yang L, Albarrán-Juárez J, et al. Tumour-cellinduced endothelial cell necroptosis via death receptor 6 promotes metastasis. Nature 2016;536:215-8.

27. Galluzzi L, Kepp O, Chan FK, et al. Necroptosis: Mechanisms and Relevance to Disease. Annu Rev Pathol 2017;12:103-30.

28. Schmidt SV, Seibert S, Walch-Rückheim B, et al. RIPK3 expression in cervical cancer cells is required for PolyICinduced necroptosis, IL-1 $\alpha$ release, and efficient paracrine dendritic cell activation. Oncotarget 2015;6:8635-47.

29. Li C, Hashimi SM, Good DA, et al. Apoptosis and microRNA aberrations in cancer. Clin Exp Pharmacol Physiol 2012;39:739-46.

30. Mathew LK, Skuli N, Mucaj V, et al. miR-218 opposes a critical RTK-HIF pathway in mesenchymal glioblastoma. Proc Natl Acad Sci U S A 2014;111:291-6.

31. Williams M, Kirschner MB, Cheng YY, et al. miR-193a- 
$3 \mathrm{p}$ is a potential tumor suppressor in malignant pleural mesothelioma. Oncotarget 2015;6:23480-95.

32. Zhou R, Zhang J, Zeng D, et al. Immune cell infiltration as a biomarker for the diagnosis and prognosis of stage I-III colon cancer. Cancer Immunol Immunother 2019;68:433-42.

33. Xu J, Dai S, Yuan Y, et al. A Prognostic Model for Colon Cancer Patients Based on Eight Signature Autophagy Genes. Front Cell Dev Biol 2020;8:602174.

34. Liang Z, Li X, Liu S, et al. MiR-141-3p inhibits cell proliferation, migration and invasion by targeting TRAF5 in colorectal cancer. Biochem Biophys Res Commun 2019;514:699-705.

35. Li X, Wang Y, Wang Y, et al. MiR-141-3p ameliorates RIPK1-mediated necroptosis of intestinal epithelial cells in necrotizing enterocolitis. Aging (Albany NY) 2020;12:18073-83.

36. Wang H, Pan JQ, Luo L, et al. NF- $\kappa$ B induces miR148 a to sustain TGF- $\beta /$ Smad signaling activation in glioblastoma. Mol Cancer 2015;14:2 .

37. Ma W, Zhang X, Chai J, et al. Circulating miR-148a is a significant diagnostic and prognostic biomarker for patients with osteosarcoma. Tumour Biol 2014;35:12467-72.

38. Kjersem JB, Ikdahl T, Lingjaerde OC, et al. Plasma microRNAs predicting clinical outcome in metastatic colorectal cancer patients receiving first-line oxaliplatinbased treatment. Mol Oncol 2014;8:59-67.

39. Liu D, Zhang H, Cui M, et al. Hsa-miR-425-5p promotes tumor growth and metastasis by activating the CTNND1mediated $\beta$-catenin pathway and EMT in colorectal cancer. Cell Cycle 2020;19:1917-27.

40. Gu C, Hou C, Zhang S. miR-425-5p improves inflammation and septic liver damage through negatively regulating the RIP1-mediated necroptosis. Inflamm Res 2020;69:299-308.

41. Hasáková K, Bezakova J, Vician M, et al. Genderdependent expression of leading and passenger strand

Cite this article as: Huang Y, Zou Y, Xiong Q, Zhang C, Sayagués JM, Shelat VG, Wang X. Development of a novel necroptosis-associated miRNA risk signature to evaluate the prognosis of colon cancer patients. Ann Transl Med 2021;9(24):1800. doi: 10.21037/atm-21-6576 of miR-21 and miR-16 in human colorectal cancer and adjacent colonic tissues. Physiol Res 2017;66:S575-82.

42. Xiao G, Tang H, Wei W, et al. Aberrant Expression of MicroRNA-15a and MicroRNA-16 Synergistically Associates with Tumor Progression and Prognosis in Patients with Colorectal Cancer. Gastroenterol Res Pract 2014;2014:364549.

43. Qian J, Jiang B, Li M, et al. Prognostic significance of microRNA-16 expression in human colorectal cancer. World J Surg 2013;37:2944-9.

44. Narayanankutty A. PI3K/ Akt/mTOR Pathway as a Therapeutic Target for Colorectal Cancer: A Review of Preclinical and Clinical Evidence. Curr Drug Targets 2019;20:1217-26.

45. Hu S, Chang X, Zhu H, et al. PI3K mediates tumor necrosis factor induced-necroptosis through initiating RIP1-RIP3-MLKL signaling pathway activation. Cytokine 2020;129:155046.

46. Ranjan A, Iwakuma T. Non-Canonical Cell Death Induced by p53. Int J Mol Sci 2016;17:2068.

47. Cho SY, Gwak JW, Shin YC, et al. Expression of Hippo pathway genes and their clinical significance in colon adenocarcinoma. Oncol Lett 2018;15:4926-36.

48. Kim A, Lee SY, Kim BY, et al. Elimination of Teratogenic Human Induced Pluripotent Stem Cells by Bee Venom via Calcium-Calpain Pathway. Int J Mol Sci 2020;21:3265.

49. PodgÓrska M, Pietraszek-Gremplewicz K, OlszaŃska J, et al. The Role of Apelin and Apelin Receptor Expression in Migration and Invasiveness of Colon Cancer Cells. Anticancer Res 2021;41:151-61.

50. Lomphithak T, Akara-Amornthum P, Murakami K, et al. Tumor necroptosis is correlated with a favorable immune cell signature and programmed death-ligand 1 expression in cholangiocarcinoma. Sci Rep 2021;11:11743.

(English Language Editor: J. Teoh) 

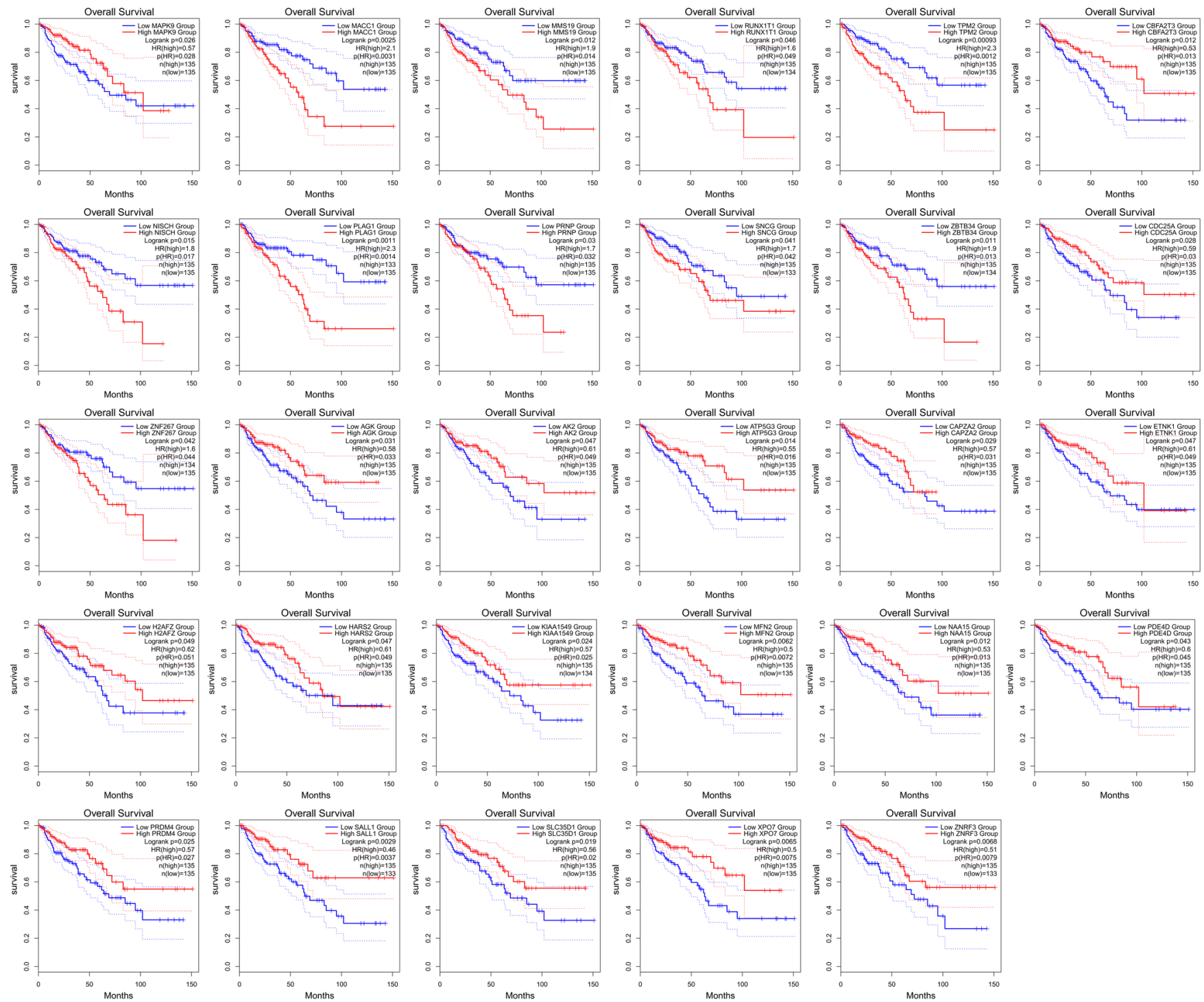

Figure S1 Partial target gene analysis of OS in COAD patients. The relationship between OS and the expression of some target genes in COAD patients was analyzed. COAD, colon adenocarcinoma; OS, overall survival. 
Table S1 Sixteen miRNAs known to be associated with necroptosis

Necroptosis-associated miRNAs
miR-495
miR-331-3p
miR-15a
miR-148a-3p
miR-7-5p
miR-141-3p
miR-425-5p
miR-200a-5p
miR-210
miR-223-3p
miR-500a-3p
miR-181-5p
miR-16-5p
miR-371-5p
miR-373
miR-543


Table S2 The primer sequences of necroptosis -related prognostic miRNAs

$$
\text { has-miR-141-3p has-miR-148a-3p }
$$

has-miR-16-5p

has-miR-200a-5p

has-miR-223-3p

has-miR-425-5p

has-miR-7-5p

reverse 5'-GTtGCTGGGAGGCTAAGATGAG-3' 5'-GCTGTCAACGATACGCTACG-3' $\quad$ 5'-GTGCAGGGTCCGAGGT-3' 5'-GTGCAGGGTCCGAGGT-3'

5'-TGGTGTCGTGGAGTCG-3'

5'-CCAGTGCAGGGTCCGAGGT-3

GAAGACTAGTGATTT-3' 5'-CGCTTCGGCAGCACATATAC-3'

Table S3 Expression of 7 miRNAs with significant expression difference between colon cancer and normal tissues

\begin{tabular}{lc}
\hline group1mea & group2mea \\
\hline 4.802798 & 11.3477 \\
12.21785 & 17.32932 \\
5.155349 & 10.69774 \\
10.0464 & 12.21582 \\
5.636136 & 9.502325 \\
7.601362 & 9.751484 \\
\hline
\end{tabular}

$\log \mathrm{FC}$

$\operatorname{logFC}$

$\mathrm{p}$

$\begin{array}{lrl}1.240452 & 1.23 \mathrm{E}-06 & 3.25 \mathrm{E}-06 \\ 0.504224 & 1.25 \mathrm{E}-06 & 3.25 \mathrm{E}-06\end{array}$

FDR

$1.23 \mathrm{E}-06$

$1.47 \mathrm{E}-05$

$2.85 \mathrm{E}-06$

$5.39 \mathrm{E}-05$

$325 \mathrm{E}-06$

0.753574

0.359364

$1.44 \mathrm{E}-06$

$5.12 \mathrm{E}-0$

$6.93 \mathrm{E}-05$

3.25E-06 